\title{
Hyaluronsäurefiller bieten die höchste Patientensicherheit
}

Minimalinvasive Behandlungen mit Hyaluronsäurefillern weisen ein besonders gutes Nutzen-Risikoprofil auf. Der große Vorteil im Vergleich zu anderen Füllmaterialien ist zudem, dass bei Überkorrektur die Wirkung mit dem Enzym Hyaluronidase antagonisiert werden kann. „Hyaluronidase ist daher in unserer Praxis ein unverzichtbarer Begleiter geworden“, erklärte Dr. Tanja C. Fischer, Ärztliche Leiterin des Haut- und Lasercentrums Pots-
dam-Berlin. Ihres Erachtens sollte das Enzym in jeder Praxis verfügbar sein, in der Hyaluronsäureinjektionen durchgeführt werden. Damit können auch Verhärtungen oder Fehlinjektionen korrigiert werden. Zudem ist es das Notfallmedikament erster Wahl bei intravasaler Injektion.

Als weitere Risikominimierung für den Patienten komme es darauf an, nur gute Produkte, die über klinische Prüfungen verfügen einzusetzen. Zudem gibt es bestimmte Indikationen wie die Glabella oder Tränenrinne, die sich nur für die Injektion mit niedrigviskösen Hyaluronsäurefillern eignen.

Monophasische polydensifizierte Hyaluronsäurefiller (z.B. Belotero ${ }^{\circ}$ ) zeichnen sich durch eine besonders gute Gewebeverträglichkeit und einfache Injizierbarkeit aus, zu deren Wirksamkeit und Verträglichkeit mehrere klinische Studien vorliegen. Die Produkte sind auch mit bereits enthaltenen Lokalanästhetika erhältlich, was die Implantation für Patienten angenehmer macht. Dr. Susanne Kammerer

Merz Aesthetics Expertentreffen „Vom Profi zum Virtuosen - Ästhetik trifft Kunst", Frankfurt/Main, 20.2.2016; Veranstalter: Merz Pharmaceuticals

\section{Multiaktive Option bei Rötungen}

PyratineXR $^{\circledast}$ ist eine multiaktive Pflege mit erwiesener Wirkung bei Rötungen, Läsionen (Papeln und Pusteln), erweiterten Äderchen und fleckiger Pigmentierung. Darüber hinaus lindert die Creme Entzündungen und erhöht das Feuchthaltevermögen der Haut durch die Wiederherstellung der Barrierefunktion. Somit hat das Präparat einen vielfältigen und breiten Anwendungsbereich bei Patienten mit unterschiedlichen Symptomen von Rosazea. Die Creme bietet mithilfe der fortgeschrittenen Cytokinin-Technologie eine effektive und gut verträgliche Langzeit-Alternative und ist auch als adjuvante Therapie einsetzbar.

Nach Daten von 12- und 48-wöchigenStudien erzeugt PyratineXR eine fortschreitende Abnahme der Symptome, die mit Rosazea verbunden sind [Tremaine AM et al. J Drugs Dermatol 2010; Ortiz A et al. J Drugs Dermatol 2009; McCullough JL et al. J Drugs Dermatol 2008]. $80 \%$ der Patienten erreichten eine Ge-

samtverbesserung nach 12 Wochen, einschließlich der Verringerung der Rötungen und Läsionen. In einer Langzeitstudie wurde die Anzahl der Läsionen nach 48 Wochen um $89 \%$ reduziert.

PyratineXR ${ }^{\circledR}$ enthält Furfuryl-Tetrahydropyranyladenin, ein patentiertes Molekül aus der Familie der Zytokinine - das sind in der Natur auftretende, pflanzliche Wachstumsregler, bei denen klinisch nachgewiesen wurde, dass sie Rötungen, Läsionen, Besenreiser und dunkle Flecken reduzieren. Die Creme ist für alle Hauttypen geeignet, sehr gut verträglich und enthält weder Parabene noch Mineralöl.

Nach Informationen von Nordesta

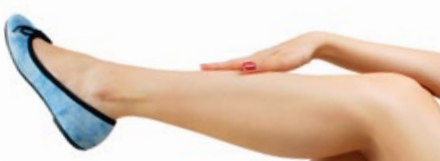

\section{Behandlung von Besenreisern und retikulären Varizen}

Mit Sklerotherapie und Laserbehandlung stehen zwei minimalinvasive Verfahren zur Beseitigung von Besenreisern und retikulären Varizen zur Verfügung. Die aktuelle Leitlinie der European Society for Laser Dermatology (ESLD) zeigt auf, warum die Sklerotherapie als First-LineTreatment zur Behandlung von C1-Varizen betrachtet wird und wo die Grenzen des Lasers liegen (Adamič M et al. J Eur Acad Dermatol Venereol 2015;29:166178). Die entscheidenden Vorteile des Ver- fahrens: Wirksamkeit und Verträglichkeit der Behandlungsmethode sind durch Studien umfassend belegt [Zhang J et al. Phlebology 2012;27:184-90; Peterson JD et al. Dermatol Surg 2012;38:1322-30]. Zudem lassen sich mithilfe der Sklerotherapie auch Besenreiser und retikuläre Varizen ausschalten, die der Laser aufgrund mangelnder Eindringtiefe nicht erreicht - darunter etwaige „Nährvenen“, von denen viele Besenreiser gespeist werden: Bleiben diese unbehandelt, verhin-
Minimalinvasive Behandlungen vereinfachen die Beseitigung von Besenreisern.

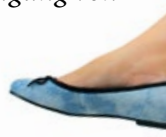

dern sie durch anhaltenden Zufluss die erfolgreiche Beseitigung von Besenreisern. Weiterhin überzeugt die Sklerotherapie durch das schnellere Eintreten sichtbarer kosmetischer Erfolge sowie die schmerzärmere Behandlung. Vertiefende Informationen finden Interessierte unter www.besenreiser-krampfaderfrei.de.

Nach Informationen von Kreussler \& Co. 